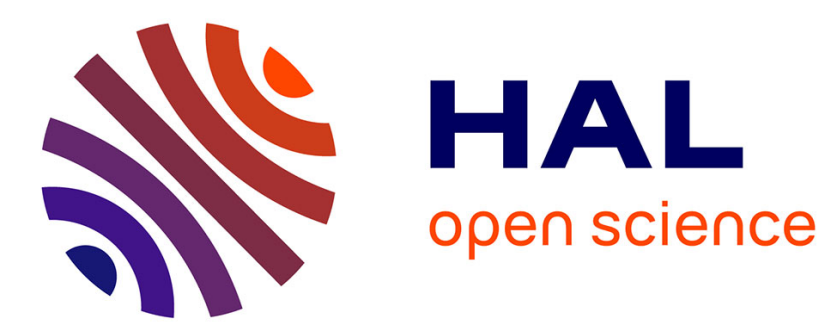

\title{
Super-convergence in maximum norm of the gradient for the Shortley-Weller method
}

\author{
Lisl Weynans
}

\section{To cite this version:}

Lisl Weynans. Super-convergence in maximum norm of the gradient for the Shortley-Weller method. [Research Report] RR-8757, INRIA Bordeaux; INRIA. 2017, pp.16. hal-01176994v3

\section{HAL Id: hal-01176994 \\ https://hal.inria.fr/hal-01176994v3}

Submitted on 28 Aug 2017

HAL is a multi-disciplinary open access archive for the deposit and dissemination of scientific research documents, whether they are published or not. The documents may come from teaching and research institutions in France or abroad, or from public or private research centers.
L'archive ouverte pluridisciplinaire HAL, est destinée au dépôt et à la diffusion de documents scientifiques de niveau recherche, publiés ou non, émanant des établissements d'enseignement et de recherche français ou étrangers, des laboratoires publics ou privés. 


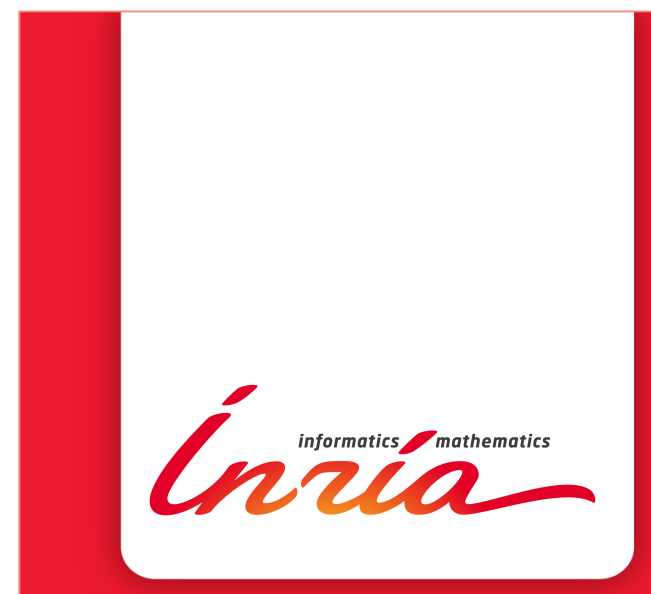

Super-convergence in maximum norm of the gradient for the Shortley-Weller method.

\section{Weynans ${ }^{1}$}

${ }^{1}$ Team Memphis, INRIA Bordeaux-Sud-Ouest \& CNRS UMR 5251,

Université de Bordeaux, France

\section{RESEARCH}

\section{REPORT}

$\mathrm{N}^{\circ} 8757$

Juillet 2017

Project-Teams Memphis 



\title{
inzián
}

\section{Super-convergence in maximum norm of the gradient for the Shortley-Weller method.}

\author{
L. Weynans ${ }^{\prime *}$ \\ ${ }^{1}$ Team Memphis, INRIA Bordeaux-Sud-Ouest \& CNRS UMR 5251, \\ Université de Bordeaux, France \\ Project-Teams Memphis \\ Research Report $\mathrm{n}^{\circ} 8757$ - Juillet 2017 - 16 pages
}

\begin{abstract}
We prove in this paper the second-order super-convergence in $L^{\infty}$-norm of the gradient for the Shortley-Weller method. Indeed, this method is known to be second-order accurate for the solution itself and for the discrete gradient, although its consistency error near the boundary is only first-order. We present a proof in the finite-difference spirit, using a discrete maximum principle to obtain estimates on the coefficients of the inverse matrix. The proof is based on a discrete Poisson equation for the discrete gradient, with second-order accurate Dirichlet boundary conditions. The advantage of this finite-difference approach is that it can provide pointwise convergence results depending on the local consistency error and the location on the computational domain.
\end{abstract}

Key-words: Finite-difference, Poisson equation, super-convergence, discrete Green's function, Shortley-Weller method

* Corresponding author: lisl.weynans@inria.fr

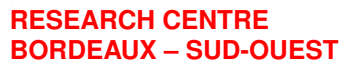

351, Cours de la Libération

Bâtiment A 29

33405 Talence Cedex 


\section{Super-convergence du gradient en norme infinie pour la méthode de Shortley-Weller.}

Résumé : Nous présentons dans ce rapport une preuve de la super-convergence à l'ordre deux du gradient, en norme $L^{\infty}$ pour la méthode de Shortley-Weller. En effet, avec cette méthode le gradient discret converge à l'ordre deux même si l'erreur de troncature près du bord du domaine est d'ordre un seulement, et que la solution elle-même ne converge aussi qu'à l'ordre deux. La preuve est réalisée avec une technique de différences finies, inspirée par l'article de Ciarlet [1, et utilisant un principe du maximum discret pour obtenir des estimations des coefficients de la matrice inverse. Elle utilise une formulation discrète de l'équation de Poisson pour le gradient discret, avec des conditions au bord de Dirichlet à l'ordre deux. Cette approche par différences finies permet d'obtenir des résultats de convergence locaux, en fonction des différentes valeurs de l'erreur de troncature et de la position du point considéré sur le domaine de calcul. Elle permet aussi d'obtenir des résultats en norme $L^{\infty}$.

Mots-clés : Différences finies, super-convergence, fonction de Green discrète, méthode de Shortley-Weller 


\section{Contents}

\begin{tabular}{|lr}
\hline 1 & Introduction \\
\hline
\end{tabular}

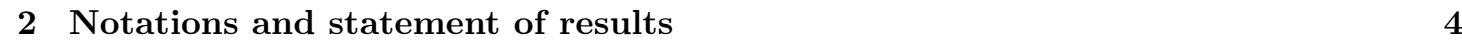

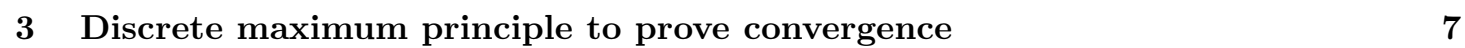

3.1 Discrete Green's function $\ldots \ldots \ldots \ldots$. . . . . . . . . . . . 7

3.2 Estimating the coefficients of the discrete Green's function . . . . . . . . . . . . . 8

$\begin{array}{|lll|}4 & \text { Reminder of the proof of high-order convergence of the solution } & 8\end{array}$

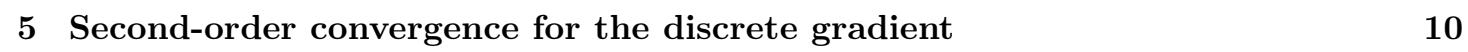

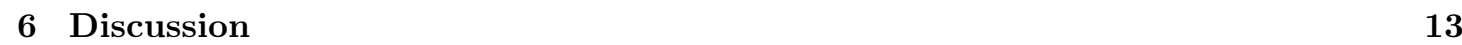

\begin{tabular}{|lr}
\hline 7 Conclusion & 14 \\
\hline
\end{tabular}

\begin{tabular}{|l|}
\hline A Numerical illustration: corners and regularity of the solution \\
\hline
\end{tabular} 


\section{Introduction}

The Shortley-Weller method is a classical finite-difference method to solve the Poisson equation with Dirichlet boundary conditions in irregular domains. It is known to converge with secondorder accuracy, although the consistency error of the numerical scheme is only first-order near the boundary. Furthermore, it has been numerically observed that the gradient of the numerical solution also converges with second-order accuracy. Recently, Yoon and Min raised in [7] the issue that mathematical justifications of this super-convergence phenomenon were lacking. Then they provided in [8, a proof of this super-convergence in a discrete $L^{2}$-norm.

Here we present a proof of the super-convergence of the gradient in a discrete $L^{\infty}$-norm, with a finite-difference technique. To our knowledge, all proofs of the super-convergence of the gradient for the Shortley-Weller method, either using finite-differences as in [8], or a finite-element formalism as in [4] and [3], have been established for discrete $L^{2}$-norms as we will discuss in $\$ 6$.

Our proof is based on the use of the discrete maximum principle, for monotone matrices, following the method presented by Ciarlet in [1]. This discrete maximum principle, applied to ad-hoc functions, leads us to obtain bounds on the coefficients of the inverse matrix. We first provide some notations, recall the Shortley-Weller method and present our results in $\$ 2$. Then we present the technique of Ciarlet [1] adapted to our case in $\$ 3$. In $\$ 4$ we recall the second-order convergence of the numerical solution in the whole domain, and the third-order convergence near the boundary. We use this property in $\$ 5$ to formulate a discrete Poisson equation for the discrete gradient, with Dirichlet boundary conditions that are second-order accurate, and we finally prove the second-order convergence of the gradient. We compare our approach to the literature in 86 .

\section{Notations and statement of results}

In the following, we consider a domain $\Omega$ belonging to $\mathbb{R}^{2}$ or $\mathbb{R}^{3}$, with a boundary $\Gamma$. The Shortley-Weller method is aimed to solve the Poisson equation in the domain $\Omega$ with Dirichlet conditions on $\Gamma$ :

$$
\left\{\begin{array}{c}
-\triangle u=f \text { in } \Omega, \\
u=g \text { on } \Gamma .
\end{array}\right.
$$

For our analysis, which is based on a finite-differences formulation, we need:

a) that a unique solution $u$ of (1) exists and is smooth enough for our consistency error analyses to be valid.

b) that the solution of problem (1) with $f=1$ and $g=0$ is $C^{1}$ near the boundary, because this property provides us estimates of the discrete Green functions in 4

Consequently, for the sake of simplicity, we assume in the whole paper that the source term $f$, the boundary $\Gamma$, and the boundary condition $g$ are such that these two properties are satisfied. In this context, the boundary $\Gamma$ may not necessarily be smooth. For instance, it may be only piecewise smooth and have corners, as soon as conditions a) and b) are satisfied. However it is known that if the boundary has corners with angles greater than a limit value, then the solution may lose its regularity near these corners. In this case, our analysis is not valid anymore. This behavior is illustrated in the appendix. Let us notice that the case of convergence when singularities occur near the interface has been handled in 3] with a finite-element approach, obtaining a $O\left(h^{1.5}\right)$ convergence in a discrete $H^{1}$ - norm.

The problem (1) is discretized on a uniform cartesian grid, see Figure 1. For the sake of clarity, the figures will represent the discretization points in two dimensions only, but the formulation of the problem and the proofs of convergence will be presented in three dimensions. The grid 

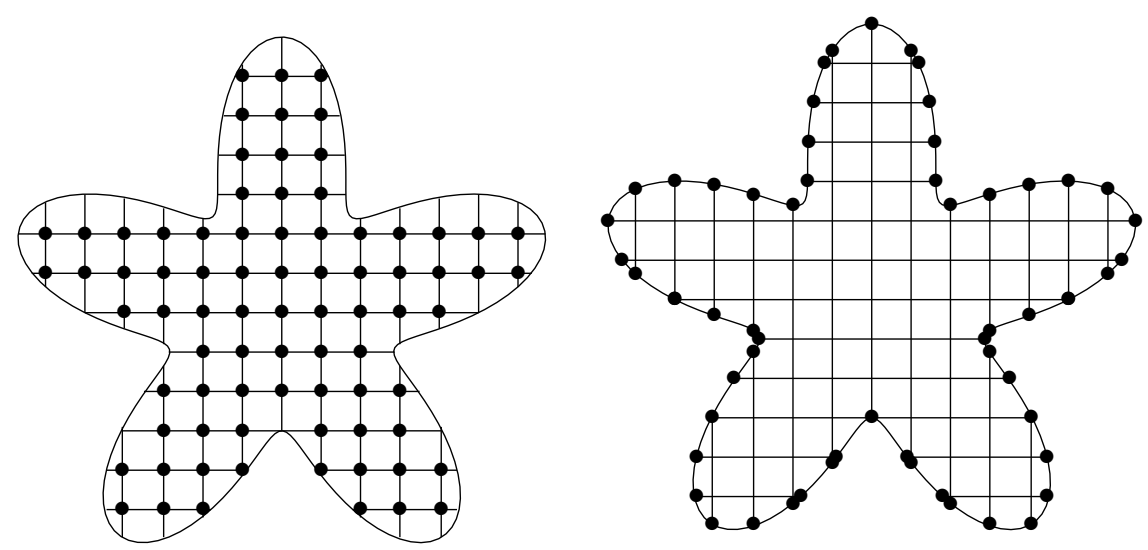

Figure 1: Left: interior nodes, belonging to $\Omega_{h}$, right: boundary nodes, belonging to $\Gamma_{h}$.
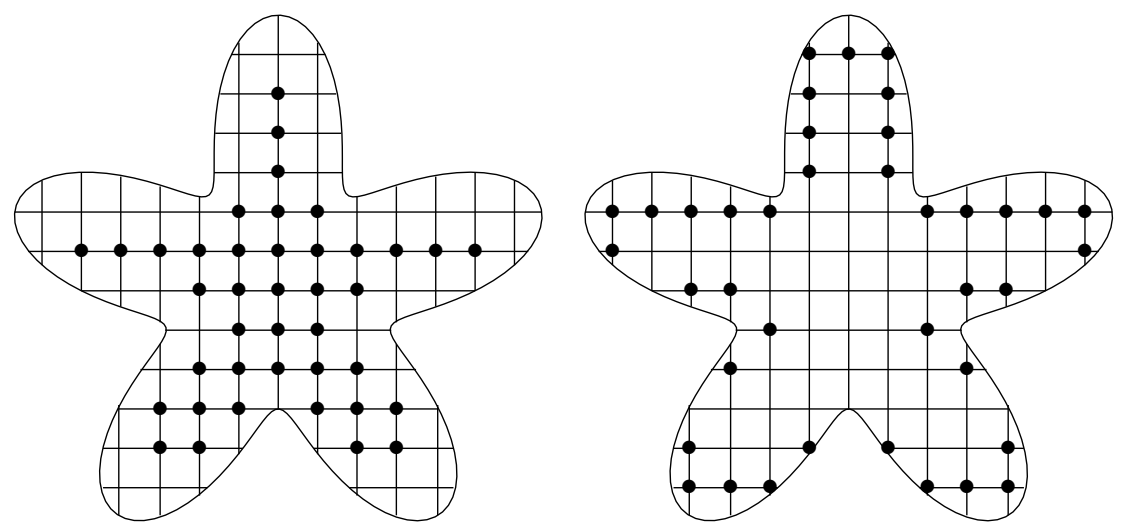

Figure 2: Left: regular nodes, i.e. belonging to $\Omega_{h}^{* *}$, right: irregular nodes, i.e. belonging to $\Omega_{h}^{*}$.

spacing is denoted by $h$, and the coordinates of the points on the grid are defined by $\left(x_{i}, y_{j}, z_{k}\right)=$ $(i h, j h, k h)$. The points on the cartesian grid are named either with letters such as $P$ or $Q$, or with letters and indices such as $M_{i, j, k}=\left(x_{i}, y_{j}, z_{k}\right)$ if we need to have informations about the location of the point.

The set of grid points located inside the domain $\Omega$ is denoted by $\Omega_{h}$. These points are called interior nodes. The set of points located at the intersection of the axes of the grid and the boundary $\Gamma$ is denoted by $\Gamma_{h}$. These points are called boundary nodes and are used for imposing the boundary conditions in the numerical scheme, see Figure 1 for an illustration.

We say that a grid node is regular if none of its direct neighbors is on the boundary $\Gamma_{h}$, and that it is irregular if at least one of its neighbors belongs to $\Gamma_{h}$. The set of regular grid nodes is denoted by $\Omega_{h}^{* *}$, and the set of irregular grid nodes is denoted by $\Omega_{h}^{*}$. See Figure 2 for an illustration. The Shortley-Weller scheme for solving the Poisson equation with Dirichlet boundary conditions is based on a dimension by dimension approach. In the following, for the sake of clarity we use the same notations as in the paper of Yoon and Min [7].

Let the six neighboring nodes of a grid node $P$ inside the domain be named as $P_{i}, \quad 1 \leq i \leq 6$ and the distances between $P$ and these nodes as $h_{i}, 1 \leq i \leq 6$. If $P$ is a regular node then $h_{i}=h$ for all $1 \leq i \leq 6$. If $P$ is an irregular node then at least one of the $h_{i}$ is different from $h$. 
The discretization of the Laplace operator with the Shortley-Weller method reads:

$$
\begin{aligned}
-\triangle_{h} u_{h}(P)= & \left(\frac{2}{h_{1} h_{2}}+\frac{2}{h_{3} h_{4}}+\frac{2}{h_{5} h_{6}}\right) u_{h}(P)-\frac{2}{h_{1}\left(h_{1}+h_{2}\right)} u_{h}\left(P_{1}\right) \\
& -\frac{2}{h_{2}\left(h_{1}+h_{2}\right)} u_{h}\left(P_{2}\right)-\frac{2}{h_{3}\left(h_{3}+h_{4}\right)} u_{h}\left(P_{3}\right)-\frac{2}{h_{4}\left(h_{3}+h_{4}\right)} u_{h}\left(P_{4}\right) \\
& -\frac{2}{h_{5}\left(h_{5}+h_{6}\right)} u_{h}\left(P_{5}\right)-\frac{2}{h_{6}\left(h_{5}+h_{6}\right)} u_{h}\left(P_{6}\right) .
\end{aligned}
$$

The matrix associated with this linear system has all its diagonal entries strictly positive, all off-diagonal entries nonpositive (or negative or zero) and is irreducibly diagonally dominant. Consequently it is a monotone matrix. Therefore, all coefficients of the inverse matrix are positive. This property will allow us to apply a discrete maximum principle useful to bound the coefficients of the inverse matrix.

We denote by $u_{h}$ the numerical solution of problem (1) with the Shortley-Weller method. The local error on a node $P$ is defined by $e_{h}(P)=u(P)-u_{h}(P)$. We denote by $\phi(P)$ the distance between a node $P$ and the boundary $\Gamma$. The following result, presented in [5] and in 8, will be useful for our purpose, because it provides second-order boundary conditions for a discrete Laplace operator applied to the components of the gradient.

Theorem 1. For the Shortley-Weller method, the local error $e_{h}(P)$ at node $P$ satisfies

$$
\begin{aligned}
& \left|e_{h}(P)\right| \leq O\left(h^{2}\right) \quad \forall P \in \Omega_{h}, \\
& \left|e_{h}(P)\right| \leq O\left(h^{2}\right)\left(\phi(P)+\min \left(h_{i}\right)\right), \quad \forall P \text { such that } \phi(P)=O(h),
\end{aligned}
$$

with $h_{i}, 1 \leq i \leq 6$ defined as above. We will briefly recall in $\$ 4$ the proof of this theorem in the formalism of discrete Green functions, in spite of its redundancy with the references above, because it will help us to introduce our notations and to present the proof of convergence of the discrete gradient.

Concerning the convergence of the gradient, in practice, we will only study the convergence of the discrete version of $\partial_{x} u$, because the $x-, y-$ and $z$-directions have symmetric behaviors.

We define $S_{h}$ as:

$$
S_{h}=\left\{P, P \text { middle of }[M N], M, N \in \Omega_{h} \cup \Gamma_{h}, M \text { and } N \text { adjacent in the x-direction. }\right\}
$$

We define the discrete $x$-derivative $D_{x} u_{h}(P)$ at every point $P$ in $S_{h}$ as

$$
D_{x} u_{h}(P)=\frac{u_{h}(M)-u_{h}(N)}{x_{M}-x_{N}}
$$

where $M$ and $N$ are the points belonging to $\Omega_{h} \cup \Gamma_{h}$ such that $P$ is defined as the middle of $[M N]$.

We divide $S_{h}$ into two new subsets of points (see Figure 3):

$$
\begin{aligned}
& \tilde{\Omega}_{h}=\left\{P \in S_{h}, \text { all direct neigbors of } P \text { in } S_{h} \text { are at distance } h \text { from } P\right\}, \\
& \tilde{\Gamma}_{h}=S_{h} \backslash \tilde{\Omega}_{h} .
\end{aligned}
$$

By construction, it is possible to apply the classical second-order seven points stencil for the Laplacian to all points belonging to $\tilde{\Omega}_{h}$. Remark also that the points in $\tilde{\Gamma}_{h}$ satisfy by construction the property

$$
\phi(P) \leq 3 h \quad \forall P \in \tilde{\Gamma}_{h} .
$$



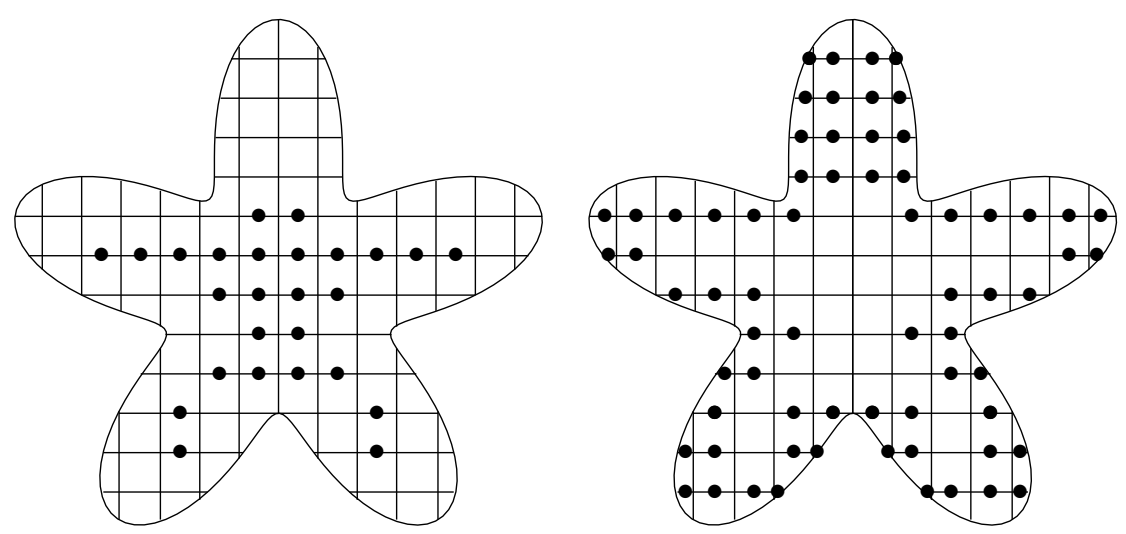

Figure 3: Left: nodes belonging to $\tilde{\Omega}_{h}$, right: nodes belonging to $\tilde{\Gamma}_{h}$

Theorem 2. For the Shortley-Weller method, the local error on the discrete $x$-derivative is second-order accurate:

$$
\left|\partial_{x} u(P)-D_{x} u_{h}(P)\right| \leq O\left(h^{2}\right), \quad \forall P \in S_{h}
$$

\section{Discrete maximum principle to prove convergence}

Here we recall the principle of the method presented in 1 to prove high-order convergence for finite-differences operators with the help of the discrete maximum principle. As we do not use exactly the same discretization matrix as in [1], due to the different way to account for boundary conditions, we present the reasoning in our case.

\subsection{Discrete Green's function}

For each $Q \in \Omega_{h}$, define the discrete Green's function $G_{h}(:, Q)=\left(G_{h}(P, Q)\right)_{P \in \Omega_{h} \cup \Gamma_{h}}$ as the solution of the discrete problem:

$$
\left\{\begin{array}{rl}
-\triangle_{h} G_{h}(:, Q)(P)=\left\{\begin{array}{cc}
0, & P \neq Q \\
1, & P=Q
\end{array}\right. \\
G_{h}(P, Q)=0, \quad P \in \Gamma_{h} .
\end{array} \quad P \in \Omega_{h},\right.
$$

In fact, each discrete Green function $G_{h}(:, Q)$ represents a column of the inverse matrix of the discrete operator $\left(-\triangle_{h}\right)$. The matrix of $\left(-\triangle_{h}\right)$ being monotone, as we noticed in $\$ 2$, it means that all values of $G_{h}(:, Q)$ are positive.

With this definition we can write the solution of the numerical problem as a sum of the source terms multiplied by the local values of the discrete Green function:

$$
u_{h}(P)=\sum_{Q \in \Omega_{h}} G_{h}(P, Q)\left(-\triangle_{h} u_{h}\right)(Q), \quad \forall P \in \Omega_{h} .
$$

In this formula we assume that $u_{h} \equiv 0$ on $\Gamma_{h}$. However, if one wants to impose non-homogeneous Dirichlet boundary conditions, it is possible to take them into account by modifying the source terms for the nodes belonging to $\Omega_{h}^{*}$. 


\subsection{Estimating the coefficients of the discrete Green's function}

Lemma 1. Let $S$ be a subset of grid nodes (thus corresponding also to a subset of the indices of the matrix), $W$ a discrete function with $W \equiv 0$ on $\Gamma_{h}$, and $\alpha>0$ such that:

$$
\left\{\begin{array}{c}
\left(-\triangle_{h} W\right)(P) \geq 0 \quad \forall P \in \Omega_{h} \\
\left(-\triangle_{h} W\right)(P) \geq \alpha^{-i} \text { for all } P \in S
\end{array}\right.
$$

Then

$$
\sum_{Q \in S} G_{h}(P, Q) \leq \alpha^{i} W(P)
$$

Proof of Lemma 1

Using the definition of the discrete Green function, we can write

$$
-\triangle_{h}\left(\sum_{Q \in S} G_{h}(:, Q)\right)(P)= \begin{cases}1 & \text { if } P \in S, \\ 0 & \text { if } P \notin S .\end{cases}
$$

Therefore,

$$
-\triangle_{h}\left(W-\alpha^{-i} \sum_{Q \in S} G_{h}(:, Q)\right)(P) \geq 0 \quad \forall P \in \Omega_{h} .
$$

As all coefficients of the inverse of $-\triangle_{h}$ are positive, it leads to

$$
W(P)-\alpha^{-i} \sum_{Q \in S} G_{h}(P, Q) \geq 0 \quad \forall P \in \Omega_{h}
$$

and finally we obtain an estimate of the coefficients of $\sum_{Q \in S} G_{h}(:, Q)$ in terms of the coefficients of $W$ :

$$
\sum_{Q \in S} G_{h}(P, Q) \leq \alpha^{i} W(P)
$$

\section{Reminder of the proof of high-order convergence of the solution}

This section is devoted to a short reminder of the proof of Theorem 1. Adequate subsets $S$ and functions $W$ are used to prove second-order convergence in $L^{\infty}$-norm in the whole numerical domain, and third-order convergence for the grid nodes whose distance to the boundary is $O(h)$.

Proof of Theorem 1 .

We denote by $\tau(P)$ the consistency error of the Shortley-Weller method on a point $P$ belonging to $\Omega_{h}$. With a classical Taylor expansion one can prove that

$$
\tau(P)=\left\{\begin{aligned}
O\left(h^{2}\right) & \text { if } P \in \Omega_{h}^{* *} \\
O(h) & \text { if } P \in \Omega_{h}^{*}
\end{aligned}\right.
$$


The local error satisfies the same linear system as the numerical solution $u_{h}(P)$, but with the consistency error as a source term:

$$
-\triangle_{h} e_{h}(P)=\tau(P) \quad \forall P \in \Omega_{h} .
$$

We consider a point $M=\left(x_{M}, y_{M}, z_{M}\right)$ inside $\Omega$. We define the discrete function:

$$
W(Q)=\frac{C-\left(x_{Q}-x_{M}\right)^{2}-\left(y_{Q}-y_{M}\right)^{2}-\left(z_{Q}-z_{M}\right)^{2}}{6},
$$

with $\left(x_{Q}, y_{Q}, z_{Q}\right)$ the coordinates of the point $Q$, and $C$ such that $W(Q) \geq 0$ for all $Q \in \Omega_{h}$. For instance we take $C=2(\operatorname{diam}(\Omega))^{2}$. We can write

$$
-\triangle_{h} W(P)=1, \quad \forall P \in \Omega_{h} .
$$

Therefore, using Lemma 1 .

$$
\sum_{Q \in \Omega_{h}^{* *}} G_{h}(P, Q) \leq \sum_{Q \in \Omega_{h}} G_{h}(P, Q) \leq W(P) \leq \frac{(\operatorname{diam}(\Omega))^{2}}{6}, \quad \forall P \in \Omega_{h} .
$$

Now we define the discrete function

$$
\tilde{W}(Q)=\left\{\begin{array}{cc}
0 & \text { if } Q \in \Gamma_{h} \\
1 & \text { otherwise }
\end{array}\right.
$$

This function satisfies

$$
\left\{\begin{array}{l}
-\triangle_{h} \tilde{W}(Q) \geq \frac{1}{h^{2}} \quad \text { if } Q \in \Omega_{h}^{*} \\
-\triangle_{h} \tilde{W}(Q)=0 \quad \text { otherwise. }
\end{array}\right.
$$

and we can directly write, using Lemma 1 .

$$
\sum_{Q \in \Omega_{h}^{*}} G_{h}(P, Q) \leq h^{2} \tilde{W}(P) \leq h^{2}, \quad \forall P \in \Omega_{h} .
$$

Combining (6) and (7), we obtain a second-order estimate of the local error on every point $P \in \Omega_{h}:$

$$
|e(P)|=\left|\sum_{Q \in \Omega_{h}} G_{h}(P, Q) \tau(Q)\right| \leq \sum_{Q \in \Omega_{h}^{* *}} G_{h}(P, Q) O\left(h^{2}\right)+\sum_{Q \in \Omega_{h}^{*}} G_{h}(P, Q) O(h) \leq O\left(h^{2}\right) .
$$

We define $V_{h}=\sum_{Q \in \Omega_{h}^{*}} G_{h}(:, Q)$. Let us consider a point $P$ in $\Omega_{h}^{*}$. The discretization of the Laplace operator with the Shortley-Weller method reads:

$$
\begin{aligned}
-\triangle_{h} V_{h}(P)= & \left(\frac{2}{h_{1} h_{2}}+\frac{2}{h_{3} h_{4}}+\frac{2}{h_{5} h_{6}}\right) V_{h}(P)-\frac{2}{h_{1}\left(h_{1}+h_{2}\right)} V_{h}\left(P_{1}\right)-\frac{2}{h_{2}\left(h_{1}+h_{2}\right)} V_{h}\left(P_{2}\right) \\
& -\frac{2}{h_{3}\left(h_{3}+h_{4}\right)} V_{h}\left(P_{3}\right)-\frac{2}{h_{4}\left(h_{3}+h_{4}\right)} V_{h}\left(P_{4}\right)-\frac{2}{h_{5}\left(h_{5}+h_{6}\right)} V_{h}\left(P_{5}\right)-\frac{2}{h_{6}\left(h_{5}+h_{6}\right)} V_{h}\left(P_{6}\right) .
\end{aligned}
$$

We assume for instance that only $P_{1}$ belongs to $\Gamma_{h}$. Consequently, $h_{1}<h, h_{i}=h$ for $2 \leq i \leq 6$ and $V_{h}\left(P_{1}\right)=0$. We can write

$$
\left(\frac{2}{h_{1} h}+\frac{4}{h^{2}}\right) V_{h}(P)=\frac{2 V_{h}\left(P_{2}\right)}{\left(h_{1}+h\right) h}+\frac{V_{h}\left(P_{3}\right)}{h^{2}}+\frac{V_{h}\left(P_{4}\right)}{h^{2}}+\frac{V_{h}\left(P_{5}\right)}{h^{2}}+\frac{V_{h}\left(P_{6}\right)}{h^{2}}+1 .
$$


Using (7) we obtain

$$
\frac{2}{h_{1} h} V_{h}(P) \leq\left(\frac{2}{h_{1} h}+\frac{4}{h^{2}}\right) V_{h}(P) \leq 7 .
$$

We can rewrite this formula as

$$
\sum_{Q \in \Omega_{h}^{*}} G_{h}(P, Q) \leq \frac{7}{2} \min \left(h_{i}\right) h .
$$

Similar inequalities can be obtained if more than one point of the stencil for $P$ belongs to $\Gamma_{h}$.

Now we consider the elliptic problem

$$
\left\{\begin{array}{c}
-\triangle u=1 \text { in } \Omega \\
u=0 \text { on } \Gamma .
\end{array}\right.
$$

We denote $v$ the solution of this problem. We have assumed in the introduction that $v$ was $C^{1}$ near the boundary. Therefore, it satisfies $v(P)=O(\phi(P))$ for points $P$ such that $\phi(P)=O(h)$.

The operator $-\Delta_{h}$ is consistent on every grid node, consequently for $h$ small enough

$$
-\triangle_{h} v(P) \geq \frac{1}{2}, \quad \forall P \in \Omega_{h} .
$$

Therefore, using Lemma 1 and restricting ourselves to $\Omega_{h}^{* *}$,

$$
\sum_{Q \in \Omega_{h}^{* *}} G_{h}(P, Q) \leq \sum_{Q \in \Omega_{h}} G_{h}(P, Q) \leq 2 v(P) \leq O(\phi(P)), \quad \forall P \text { such that } \phi(P)=O(h) .
$$

Finally, combining 10 and 8 we can write for all points $P$ such that $\phi(P)=O(h)$

$$
\begin{aligned}
\left|e_{h}(P)\right| & =\left|\sum_{Q \in \Omega_{h}} G_{h}(P, Q) \tau(Q)\right| \leq\left|\sum_{Q \in \Omega_{h}^{* *}} G_{h}(P, Q) O\left(h^{2}\right)\right|+\left|\sum_{Q \in \Omega_{h}^{*}} G_{h}(P, Q) O(h)\right|, \\
& \leq O\left(h^{2}\right)\left(\phi(P)+\min \left(h_{i}\right)\right) .
\end{aligned}
$$

\section{Second-order convergence for the discrete gradient}

This section is devoted to the proof of Theorem 2. We aim to prove that the numerical gradient defined by (3) converges with second-order accuracy in $L^{\infty}$-norm.

Proof of Theorem 2 :

The proof is divided into two parts:

- First we prove that the discrete gradient is second-order accurate for every point $P$ in $\tilde{\Gamma}_{h}$,

- then we prove that the discrete gradient is second-order accurate for all points $P$ in $S_{h}$ by means of a discrete Laplace equation applied to it.

We know from $\$ 4$ that $e_{h}$, error for the numerical solution computed with the Shortley-Weller method, satisfies

$$
\left|e_{h}(Q)\right| \leq O\left(h^{2}\right)\left(\phi(Q)+\min \left(h_{i}\right)\right), \quad \forall Q \in \Omega_{h} \text { such that } \phi(Q) \leq 4 h,
$$


with $h_{i}, 1 \leq i \leq 6$, the distances between the node $Q$ and its direct neighbors in each direction. Because of (4), all points involved in the definition of the discrete gradient for points belonging to $\tilde{\Gamma}_{h}$ are at a distance to the boundary smaller than $4 h$.

Let us consider a point $P$ belonging to $\tilde{\Gamma}_{h}$. We denote by $M$ and $N$ the points such that $P$ is their middle. To get estimates on the discrete $x$-derivative on $P$, one has to consider two possibilities:

- One of them belongs to $\Gamma_{h}$ :

Let us assume for instance that $M$ belongs to $\Gamma_{h}$ and $N$ belongs to $\Omega_{h}$. In this case, we can write that $\phi(N) \leq\left|x_{M}-x_{N}\right|$. It means that

$$
\begin{aligned}
e_{h}(M) & =0, \\
\left|e_{h}(N)\right| & \leq O\left(h^{2}\right)\left(\phi(N)+\min \left(h_{i}\right)\right) \leq O\left(h^{2}\right)\left(\left|x_{M}-x_{N}\right|+\min \left(h_{i}\right)\right) .
\end{aligned}
$$

We use these estimates to bound the discrete $x$-derivative $D_{x} u_{h}(P)$ :

$$
\begin{aligned}
\left|\partial_{x} u(P)-D_{x} u_{h}(P)\right| & =\left|\frac{u(M)-u(N)}{x_{M}-x_{N}}+O\left(x_{M}-x_{N}\right)^{2}-\frac{u_{h}(M)-u_{h}(N)}{x_{M}-x_{N}}\right|, \\
& \leq\left|\frac{e_{h}(M)-e_{h}(N)}{x_{M}-x_{N}}\right|+O\left(x_{M}-x_{N}\right)^{2} \\
& \leq\left|\frac{O\left(h^{2}\right)\left(\left|x_{M}-x_{N}\right|+\min \left(h_{i}\right)\right)}{x_{M}-x_{N}}\right|+O\left(x_{M}-x_{N}\right)^{2} .
\end{aligned}
$$

Moreover, because $h_{i} \leq\left|x_{M}-x_{N}\right| \leq h$ for all $i=1, \ldots, 6$, for the $h_{i}$ corresponding to the node $N$, we conclude that

$$
\left|\partial_{x} u(P)-D_{x} u_{h}(P)\right| \leq O\left(h^{2}\right)
$$

- Both of them belong to $\Omega_{h}$ :

In this case, $x_{M}-x_{N}=h$ and we have

$$
\begin{gathered}
\left|e_{h}(M)\right| \leq O\left(h^{3}\right), \\
\left|e_{h}(N)\right| \leq O\left(h^{3}\right) .
\end{gathered}
$$

We can again write

$$
\begin{aligned}
\left|\partial_{x} u(P)-D_{x} u_{h}(P)\right| & \leq\left|\frac{e_{h}(M)-e_{h}(N)}{x_{M}-x_{N}}\right|+O\left(x_{M}-x_{N}\right)^{2}, \\
& \leq\left|\frac{O\left(h^{3}\right)}{h}\right|+O\left(h^{2}\right), \\
& \leq O\left(h^{2}\right) .
\end{aligned}
$$

Therefore, if $P$ belongs to $\tilde{\Gamma}_{h}$, then the discrete $x$-derivative $D_{x} u_{h}(P)$ is a second-order accurate approximation of the $x$-derivative of $u$ at point $P$ :

$$
\left|\partial_{x} u(P)-D_{x} u_{h}(P)\right| \leq O\left(h^{2}\right), \quad \forall P \in \tilde{\Gamma}_{h} .
$$


Now we consider a point $P$ belonging to $\tilde{\Omega}_{h}$. Let $M$ and $N$ be the points such that $P$ is their middle. We apply the discrete $x$-derivative to the formula of the discrete elliptic operator on the points $M$ and $N$ :

$$
D_{x}\left(-\triangle_{h} u_{h}\right)(P)=\frac{\left(-\triangle_{h} u_{h}\right)(M)-\left(-\triangle_{h} u_{h}\right)(N)}{x_{M}-x_{N}} .
$$

Because $u_{h}$ is the numerical solution of the linear system (1), we know that

$$
D_{x}\left(-\triangle_{h} u_{h}\right)(P)=D_{x} f(P), \quad \forall P \text { in } \tilde{\Omega}_{h} .
$$

The node $P$ belongs to $\tilde{\Omega}_{h}$, which means that in each direction the direct neighbors of $P$ in $S_{h}$ are at the same distance $h$ from $P$. The discrete operator $-\triangle_{h}$ applied to points belonging to $\tilde{\Omega}_{h}$ thus reduces to the classical second-order seven-point stencil. Consequently, on this point, the discrete operators $-\triangle_{h}$ and $D_{x}$ commute, and we can write

$$
D_{x}\left(-\triangle_{h} u_{h}\right)(P)=-\triangle_{h}\left(D_{x} u_{h}\right)(P), \quad \forall P \in \tilde{\Omega}_{h} .
$$

Consequently, the array $v_{h}=\left(D_{x} u_{h}(P)\right)_{P \in S_{h}}$ satisfies the linear system

$$
\begin{aligned}
-\triangle_{h} v_{h}(P) & =D_{x} f(P) \quad \forall P \in \tilde{\Omega}_{h}, \\
v_{h}(P) & =D_{x} u_{h}(P) \quad \forall P \in \tilde{\Gamma}_{h},
\end{aligned}
$$

which is a discrete version of the Laplace operator applied to the $x$ - derivative of $u$ solution of (1). The consistency errors for this linear system are the following:

- The discretization of the Laplace operator 13 has the consistency error $\tau(P)=O\left(h^{2}\right)$ for all nodes belonging to $\tilde{\Omega}_{h}$, because the Shortley-Weller scheme reduces for these nodes to the classical centered seven-points formula, and because $D_{x} f(P)$ is a second-order approximation of the $x$-derivative of $f$ at point $P$.

- The formula 14 has the consistency error $\tau(P)=O\left(h^{2}\right)$ because we know from 12 that $D_{x} u_{h}(P)$ is a second-order accurate approximation of the $x$-derivative of $u$ at a point $P$ in $\tilde{\Gamma}_{h}$.

The rows of the matrix associated with this linear system correspond either to the discretization of the Laplacian operator $(13)$, or the identity (14). This matrix has therefore all its diagonal terms strictly positive, all off-diagonal entries nonpositive (or negative or zero) and is irreducibly diagonally dominant. Consequently it is a monotone matrix.

We apply the same reasoning as in $\$$ to obtain estimates on the coefficients of the inverse matrix. As in the previous subsection, we denote by $G_{h}(:, Q)=\left(G_{h}(P, Q)\right)_{P \in \tilde{\Omega}_{h} \cup \tilde{\Gamma}_{h}}$ the column of the inverse matrix of the linear system (13)-(14) corresponding to a point $Q$ belonging to $\tilde{\Omega}_{h} \cup \tilde{\Gamma}_{h}$.

We consider a point $M=\left(x_{M}, y_{M}, z_{M}\right)$ inside $\Omega$. We define the discrete function on all points $P$ in $S_{h}$ :

$$
W(P)=\frac{C-\left(x_{P}-x_{M}\right)^{2}-\left(y_{P}-y_{M}\right)^{2}-\left(z_{P}-z_{M}\right)^{2}}{6},
$$

with $\left(x_{P}, y_{P}, z_{P}\right)$ the coordinates of the point $P$, and $C$ such that $W(Q) \geq 1$ for all $Q \in \Omega_{h}$. For instance we take $C=2(\operatorname{diam}(\Omega))^{2}+6$. We can write

$$
\begin{aligned}
&-\triangle_{h} W(P)=1, \quad \forall P \in \tilde{\Omega_{h}}, \\
& W(P) \geq 1, \quad \forall P \in \tilde{\Gamma_{h}} .
\end{aligned}
$$


Therefore, using Lemma 1 .

$$
\sum_{Q \in \tilde{\Omega}_{h} \cup \tilde{\Gamma_{h}}} G_{h}(P, Q) \leq W(P) \leq \frac{(2 \operatorname{diam}(\Omega))^{2}+6}{6}, \quad \forall P \in S_{h} .
$$

Therefore, the expression on the local error for the discrete $x$-derivative on a node $P$ belonging to $S_{h}$ reads

$$
\left|\partial_{x} u(P)-D_{x} u_{h}(P)\right|=\left|\sum_{Q \in \tilde{\Omega}_{h} \cup \tilde{\Gamma_{h}}} G_{h}(P, Q) \tau(Q)\right| \leq \frac{(2 \operatorname{diam}(\Omega))^{2}+6}{6} O\left(h^{2}\right), \quad \forall P \in S_{h} .
$$

Consequently

$$
\left|\partial_{x} u(P)-D_{x} u_{h}(P)\right| \leq O\left(h^{2}\right), \quad \forall P \in S_{h}
$$

which proves that the numerical gradient converges with second-order accuracy in $L^{\infty}{ }_{-n o r m}$.

\section{Discussion}

This work was originally motivated by the remark in the paper of Yoon and Min [6] about the lack of mathematical analysis about the super-convergence of the Shortley-Weller method. This paper was followed by [8] where the autors provided a proof of this super-convergence in a discrete $L^{2}$-norm, using a discrete divergence theorem.

To our knowledge, few other works in the literature have studied the super-convergence of the gradient for elliptic finite-difference schemes, among them [2], [4] and [3].

In 22 Ferreira and Grigorieff deal with more general elliptic operators, with variable coefficients and mixed derivatives, and prove second-order convergence in $H^{1}$ norm. The proof uses negative norms and is based on the fact that the finite difference scheme is a certain non-standard finite element scheme on triangular grids combined with a special form of quadrature.

In 4] $\mathrm{Li}$ et al. study the super-convergence of solution derivatives for the Shortley-Weller method for Poisson's equation, considering also this method as a special kind of finite element method. They obtained second-order convergence in $H^{1}$ norm for rectangular domains, and an order 1.5 for polygonal domains. The work in [3] adresses the case of unbounded derivatives near the boundary $\Gamma$, on polygonal domains.

Our approach differs from the latter because we do not use a finite-element approach. Instead we propose a proof based on a finite-difference analysis, which is a variant of the method presented in [1: we use a discrete maximum principle to obtain estimates on the coefficients of the inverse matrix, but in our case the bound on the coefficients can also vary with the rows of the inverse matrix. This variant is useful to obtain a specific bound for points located near the boundary and obtain the third-order convergence of the solution at these points, already presented in [5] and in [8. This intermediate result leads us to formulate a discrete Poisson equation for the discrete gradient, with Dirichlet boundary conditions that are second-order accurate. Then the same maximum-principle methodology is applied to the discrete gradient, leading to second-order accuracy.

The approach developed in this paper has the advantage to be simple to carry out, and to be able to provide locally pointwise estimates, instead of the usual convergence results in the discrete $L^{2}$ - or $H^{1}$-norms. 


\section{Conclusion}

We have proven that the discrete gradient obtained by the Shortley-Weller method for the Poisson equation converges with second-order accuracy in $L^{\infty}$-norm. This is a super-convergence property because the numerical solution itself converges only with second order accuracy in $L^{\infty}$-norm. This property is proven with a variant of Ciarlet's technique to obtain high-order convergence estimates for monotone finite-differences matrices. With carefully chosen test functions we are able to bound the coefficients of the discrete Green functions associated with the matrix of the Shortley-Weller method. One key ingredient is the discrete gradient as the solution of another Poisson equation with Dirichlet boundary conditions that have a second-order accuracy. A further development would be to extend this work to the case of more general elliptic operators.

\section{A Numerical illustration: corners and regularity of the so- lution}

We illustrate here the possible loss of regularity of the solution in the case of corners, that was evocated in $\$ 2$. Depending on the angles of this corners, the solution can indeed be less than $C^{1}$ near the boundary, even if the source term and the boundary conditions are very smooth.

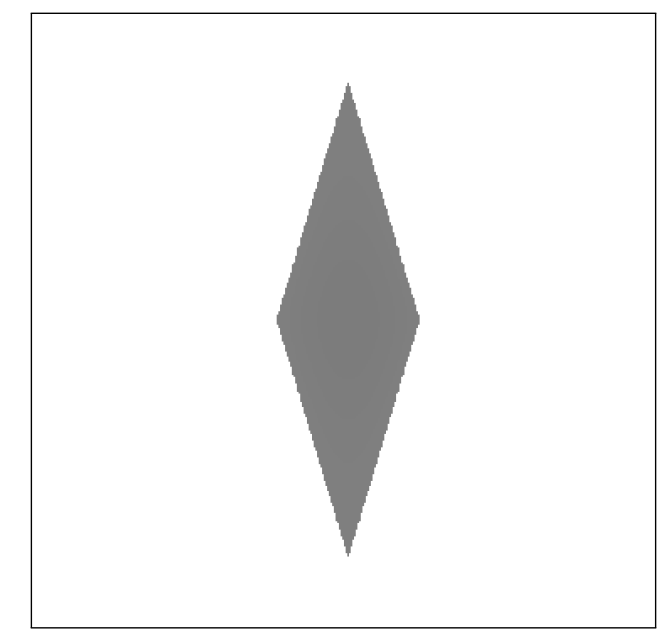

Figure 4: Exemple of domains with corners

On figure 4, we consider two domains that are only piecewise smooth: the diamond-shaped one, denoted by $\Omega_{1}$, and its complementary, denoted by $\Omega_{2}$. The angles of the first one do not exceed the value $\pi$, while some of the second one do actually.

We solve numerically the following problem

$$
\left\{\begin{array}{c}
-\triangle u=1 \text { on } \Omega, \\
u=0 \text { on } \Gamma .
\end{array}\right.
$$

in these two domains: $\Omega=\Omega_{1}$ and $\Omega=\Omega_{2}$, with the Shortley-Weller method. The numerical solution is in fact the sum of all the discrete Green functions associated to grid points in the numerical domain. In this paper, to obtain the estimate 10 on the discrete Green function, we make the assumption that the solution $u$ of problem (17) is at least $C^{1}$ near the boundary, so that it satisfies $u(x)=O(h)$ for points located at a distance $O(h)$ of the domain boundary. 
We compute in both cases, the $L^{\infty}$-norm of the numerical solution on grid points in $\Omega_{h}^{*}$ (that is, irregular grid points). The Tables 1 and 2 present these results. We observe that for domain $\Omega_{1}$, the numerical solution on $\Omega_{h}^{*}$ converges to zero at order one. For domain $\Omega_{2}$, the convergence order is strictly smaller than one, which means that the discrete Green functions do not satisfy the property that we need for our convergence estimates.

\begin{tabular}{|c|c|c|}
\hline $\mathrm{N}$ & $L^{\infty}$ norm & Convergence order \\
\hline 100 & $4.80 \mathrm{E}-003$ & - \\
200 & $2.45 \mathrm{E}-003$ & 0.970 \\
400 & $1.26 \mathrm{E}-003$ & 0.965 \\
600 & $8.43 \mathrm{E}-004$ & 0.971 \\
800 & $6.34 \mathrm{E}-004$ & 0.973 \\
1000 & $5.077 \mathrm{E}-004$ & 0.976 \\
\hline
\end{tabular}

Table 1: Convergence to zero for irregular grid points for problem 17 in domain $\Omega_{1}$.

\begin{tabular}{|c|c|c|}
\hline $\mathrm{N}$ & $L^{\infty}$ norm & Convergence order \\
\hline 100 & $1.462 \mathrm{E}-002$ & - \\
200 & $8.104 \mathrm{E}-003$ & 0.856 \\
400 & $4.503 \mathrm{E}-003$ & 0.853 \\
600 & $3.195 \mathrm{E}-003$ & 0.851 \\
800 & $2.506 \mathrm{E}-003$ & 0.850 \\
1000 & $2.075 \mathrm{E}-003$ & 0.850 \\
\hline
\end{tabular}

Table 2: Convergence to zero for irregular grid points for problem 17 in domain $\Omega_{2}$.

\section{References}

[1] P.G. Ciarlet. Discrete maximum principle for finite differences operators. Aequat. Math., 4:338-352, 1970.

[2] J.A. Ferreira and R.D. Grigorieff. On the supraconvergence of elliptic finite difference schemes. Applied Num. Math., 28:275-292, 1998.

[3] Z.-C. Li, H.-Y. Hu, S.T. Wang, and Q. Fang. Superconvergence of solution derivatives for the shortley-weller difference approximation to poisson's equation with singularities on polygonal domains. Applied Num. Math., 58:689-704, 2008.

[4] Z.-C. Li, T. Yamamoto, and Q. Fang. Superconvergence of solution derivatives for the shortley-weller difference approximation of poisson's equation. part i: smoothness problems. J. Comp. Applied Math., 151:307-333, 2003.

[5] M. Matsunaga and T. Yamamoto. Superconvergence of the shortley-weller approximation for dirichlet problems. J. Comp. Applied Math., 116:263-273, 2000.

[6] G. Yoon and C. Min. A review of the supra-convergences of shortley-weller method for poisson equation. J. KSIAM, 18(1):51-60, 2014. 
[7] G. Yoon and C. Min. Analyses on the finite difference method by gibou et al. for the poisson equation. J. Comput. Phys., 280:184-194, 2015.

[8] G. Yoon and C. Min. Convergence analysis of the standard central finite difference method for poisson equation. J Sci Comput, 67:602-617, 2016. 


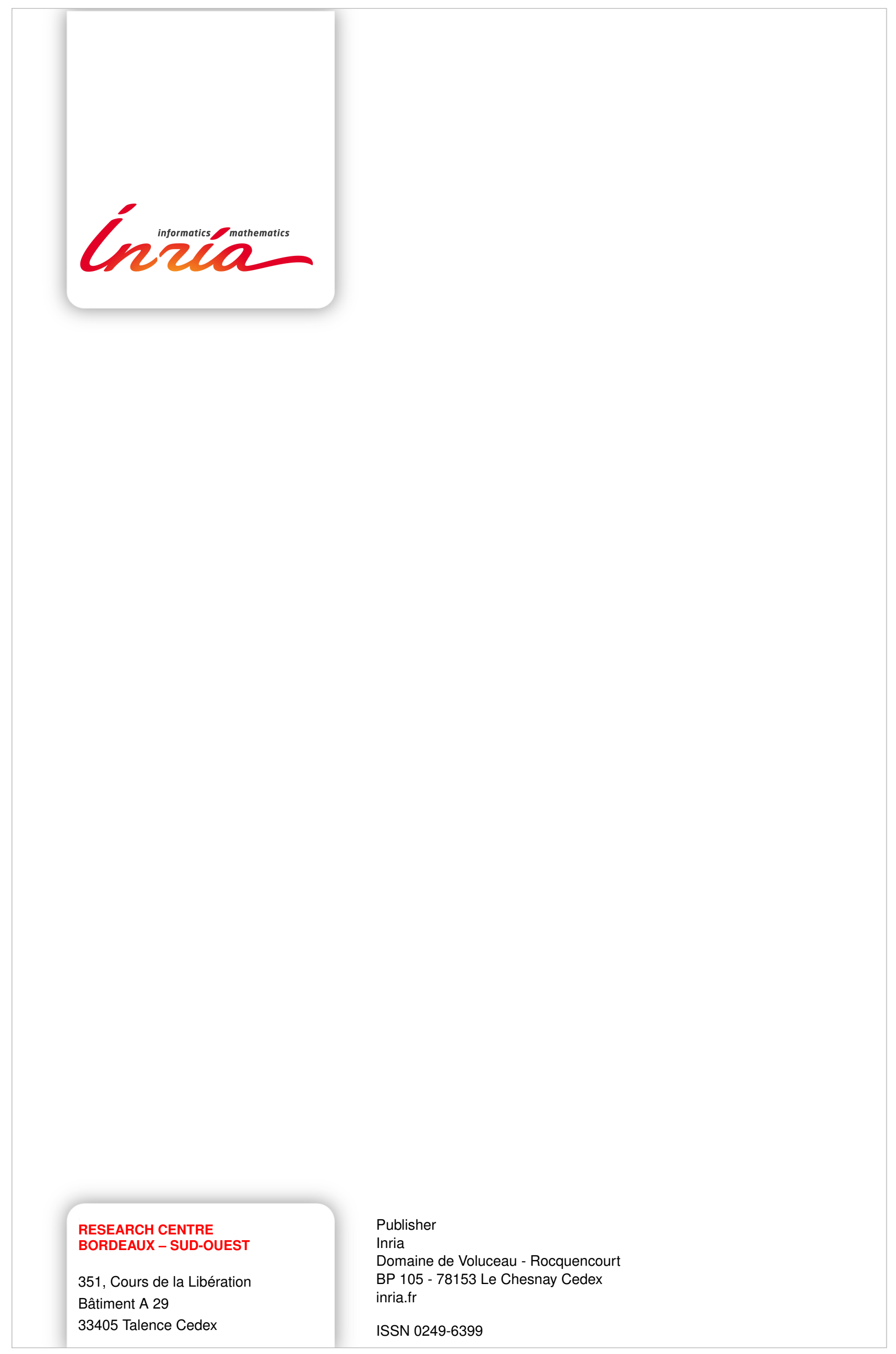

